\title{
Maximizing the Delivery of MPR Broadcasting Under Realistic Physical Layer Assumptions
}

\author{
François Ingelrest ${ }^{1}$ and David Simplot-Ryl ${ }^{2}$ \\ ${ }^{1} L C A V, E P F L, C H-1015$, Lausanne, Switzerland \\ ${ }^{2}$ IRCICA/LIFL, CNRS UMR 8022, Univ. Lille 1, INRIA Lille-Nord Europe, France
}

E-mail: Francois.Ingelrest@epfl.ch; David.Simplot@lifl.fr

Revised February 29, 2008.

\begin{abstract}
It is now commonly accepted that the unit disk graph used to model the physical layer in wireless networks does not reflect real radio transmissions, and that a more realistic model should be considered for experimental simulations. Previous work on realistic scenarios has been focused on unicast, however broadcast requirements are fundamentally different and cannot be derived from the unicast case. Therefore, the broadcast protocols must be adapted in order to still be efficient under realistic assumptions. In this paper, we study the well-known multipoint relay broadcast protocol (MPR), in which each node has to choose a set of 1-hop neighbors to act as relays in order to cover the whole 2-hop neighborhood. We give experimental results showing that the original strategy used to select these multipoint relays does not suit a realistic model. On the basis of these results, we propose new selection strategies solely based on link quality. One of the key aspects of our solutions is that our strategies do not require any additional hardware and may be implemented at the application layer, which is particularly relevant to the context of ad hoc and sensor networks where energy savings are mandatory. We finally provide new experimental results that demonstrate the superiority of our strategies under realistic physical assumptions.
\end{abstract}

Keywords ad hoc network, multipoint relay broadcasting, realistic physical layer, sensor network

\section{Introduction and Motivation}

Although wireless networking is now essential in everyday's life, the most deployed technology is still the WiFi technology. While it ensures reliable wireless communications, it is very restrictive since users must stay close to fixed access points. For the purpose of overcoming such issues, wireless ad hoc networks have been broadly studied during the past few years. They are formed by autonomous devices which operate in a selforganized manner and communicate together using radio interfaces. In such networks, because of the path loss of radio communications, only close hosts may directly communicate to each other. Long-distance communications require messages to be forwarded by multiple intermediate nodes.

Along with routing, broadcasting is one of the most important communication tasks in those networks, as it is used for many purposes (e.g., route discovery, synchronization). In a straightforward solution to broadcasting, hosts blindly relay packets upon first reception to their neighborhood, leading to a full coverage of the network (providing, of course, that the latter is connected). However, due to physical phenomena, this solution leads to the well-known broadcast storm problem $^{[1]}$. Moreover, this is a totally inefficient algorithm, since most of the retransmissions are redundant and not needed to ensure the delivery of the packet, leading to a huge amount of wasted energy.

Almost all of the alternative broadcast schemes have always been studied under ideal scenario, where the unit disk graph is used to model wireless communications. In this model, two hosts can communicate with each other if the distance between them is no more than a given communication radius. Packets never get lost and are always received without any error. This model has recently been more and more criticized since it does not correctly reflect the behavior of radio transmissions in a real environment ${ }^{[2]}$. Indeed, signal strength fluctuations have a significant impact on performance, and thus cannot be ignored in designing communication protocols.

In this paper, we focus on the well-known multipoint relay broadcast protocol $(\mathrm{MPR})^{[3]}$, used for broadcasting in ad hoc networks. We consider its default behavior under a more realistic scenario where the probability of correct reception of a packet smoothly decreases with the distance between the emitter and the receiver(s).

Regular Paper 
To achieve this, we replace the unit disk graph model with the lognormal shadowing model ${ }^{[4]}$. Since the experimental results demonstrate the needs for a more suitable algorithm, we propose several modifications to MPR in order to improve it by maximizing the delivery of the broadcast packet.

One of the key aspects of our solutions is that they highly fit ad hoc networks since they do not rely on any other specific hardware, and may be applied to any kind of wireless devices. All the modifications to MPR that we propose are indeed entirely based on link quality between neighboring nodes, which may be easily evaluated by software methods. We also show by simulations that while our solutions do not imply structural changes to the MPR algorithm, they provide very good results under the considered realistic scenario, actually they are better than the original MPR protocol.

The remainder of this paper is organized as follows. In the next section we provide the needed network definitions and then in Section 3 we present a detailed description of MPR broadcasting. In Section 4, we present the lognormal shadowing model that we use throughout this paper. In Section 5, we provide an analysis of the behavior of the original algorithm used in MPR with the realistic physical layer. We then describe in Section 6 some original solutions that better fit the realistic scenario. We finally conclude in Section 7 and give some directions for future work.

Preliminary version of this paper appeared in [5].

\section{Preliminaries}

The common representation of a wireless network is a graph $G=(V, E)$, where $V$ is the set of vertices (the hosts, or the nodes of the network) and $E \subseteq V^{2}$ the set of edges which represents the available communication links: there exists an ordered pair $(u, v) \in E$ if the node $v$ is able to physically receive packets sent by $u$ (e.g., in a single-hop fashion). The neighborhood set $N(u)$ of the node $u$ is defined as:

$$
N(u)=\{v \in V \mid v \neq u \wedge(u, v) \in E\} .
$$

The size of this set, $|N(u)|$, is also known as the degree of node $u$. The density $d$ of the network is the average degree of the nodes. To distinguish between nodes, each of them must be assigned a unique identifier which may be any arbitrary value (e.g., a MAC or an IP address).

We assume that nodes are aware of the existence of each neighboring node within a distance of 2 hops (we call this a 2-hop knowledge). In ad hoc networks, the neighborhood discovery is generally done by small con- trol messages (i.e., the well-known HELLO messages) which are regularly sent by each host. A 2-hop knowledge may easily be acquired thanks to two rounds of exchanges: nodes can indeed insert the identifiers of their neighbors into their own beacon messages.

\section{MPR Broadcasting Protocol}

As aforementioned, the easiest way to broadcast a packet is to have all the nodes forward it at least once to their neighborhood: this method is known as the blind flooding. However, such a simple behavior has a lot of drawbacks, among which we can cite the high energy consumption. Many other alternative solutions have been proposed, and an extensive review of them can be found in [6]. They may be classified into two categories.

- Centralized algorithms, that require a global knowledge of the network to be applied to (i.e., each existing node and communication links).

- Localized algorithms, that only require nodes to maintain local knowledge about their spatially nearby network nodes (their neighbors).

Obviously, localized message forwarding is a resource-efficient communication paradigm which is well tailored to ad hoc networks due to their decentralized architecture. Among all these localized solutions, we chose to focus on the multipoint relay protocol $(\mathrm{MPR})^{[3]}$ for several reasons.

- It is efficient using the unit disk graph model.

- It is used in the well-known standardized routing protocol OLSR ${ }^{[7]}$.

- It may be used for other miscellaneous purposes (e.g., computing connected dominating sets ${ }^{[8]}$ ).

In this algorithm, it is assumed that the nodes have a 2-hop knowledge: they are aware of their neighbors (1-hop distance), and the neighbors of these neighbors (2-hop distance). Its principle is as follows. Each node $u$ that has to relay the message must first elect some of its 1-hop neighbors to act themselves as relays, in order to reach the 2-hop neighbors of $u$. The selection is then forwarded within the packet and receivers can thus determine if they have been selected or not: each node that receives the message for the first time checks if it has been designated as a relaying node by the sender, and if so the message is forwarded after the selection of a new relaying set of neighbors. A variant exists where nodes proactively select their relays before having to broadcast a packet, and selection is sent within HELLO messages.

Obviously, the tricky part of this protocol lies in the selection of the set of relays $M P R(u)$ within the 1-hop neighbors of a node $u$ : the smaller this set is, the smaller 
the number of retransmissions is and the more efficient the broadcast is. Unfortunately, finding such a set that is the smallest possible one is an NP-complete problem (it is equivalent to the well-known minimum set cover $\left.^{[9]}\right)$. A greedy heuristic, which can be found in [10], is proposed by Qayyum et al. as a solution to this problem. Considering a node $u$, it can be described as follows.

1) Set $\operatorname{MPR}(u)=\emptyset$. Place all 2-hop neighbors (considering only outgoing links) in a set $M P R^{\prime}(u)$ of uncovered 2-hop neighbors.

2) While there exists a 1-hop neighbor $v$ which is the only common neighbor of $u$ and some nodes in $M P R^{\prime}(u)$ : add $v$ to $M P R(u)$, remove its neighbors from $M P R^{\prime}(u)$.

3) While the set $M P R^{\prime}(u)$ is not empty, repeatedly choose the 1-hop neighbor $v$ not present in $\operatorname{MPR}(u)$ that covers the greatest number of nodes in $M P R^{\prime}(u)$. Each time a new node is added to $\operatorname{MPR}(u)$, remove its neighbors from $M P R^{\prime}(u)$. In the case of tie, choose the node with the highest degree.

There exists a variant in the third step that was introduced in the latest version of MPR, and that may lead to the removal of a few redundant relays. Indeed, due to the greedy nature of this heuristic, adding a new relay to $\operatorname{MPR}(u)$ may make useless the previously selected ones. Thus, after each new addition, one should check if all the other relays are still really needed. This extra step greatly increases the complexity of computation since all subsets of the relays need to be considered. Moreover, it does not bring a really noticeable improvement, and the removal of redundant relays further decreases the probability of delivery when a realistic physical model is considered. For all these reasons, we choose not to consider this extra step in the remaining of this paper.

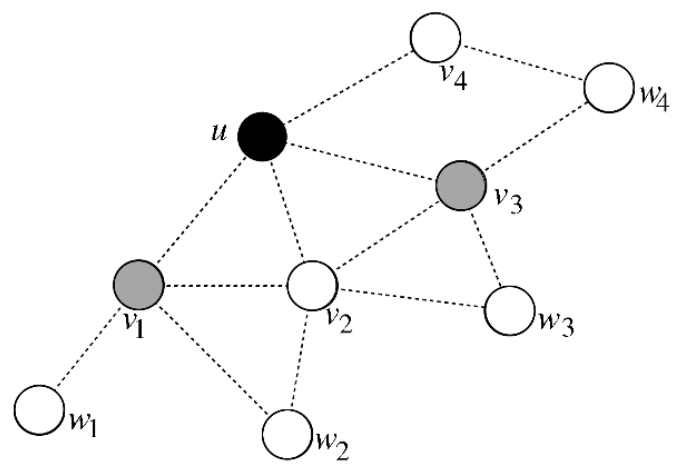

Fig.1. Applying MPR broadcasting at node $u$ : $M P R(u)=$ $\left\{v_{1}, v_{3}\right\}$.

An example of this heuristic is given in Fig.1, starting with $\operatorname{MPR}(u)=\emptyset$. The node $v_{1}$ is the only one able to reach $w_{1}$, so it is added to $\operatorname{MPR}(u)$, and nodes $w_{1}$ and $w_{2}$ are removed from $M P R^{\prime}(u)$. No other mandatory 1-hop neighbor of $u$ exists, so the other relays are selected according to the number of nodes in $\operatorname{MPR}^{\prime}(u)$ they cover. Nodes $v_{2}$ and $v_{4}$ cover only one node in $\operatorname{MPR}^{\prime}(u)$, while node $v_{3}$ covers at the same time $w_{3}$ and $w_{4}$, so $v_{3}$ is chosen and added to $\operatorname{MPR}(u)$. With the set $M P R^{\prime}(u)$ being empty, no other nodes are selected. We finally have $\operatorname{MPR}(u)=\left\{v_{1}, v_{3}\right\}$.

Being the broadcast protocol used in OLSR, MPR has been the subject of miscellaneous studies since its publication. For example in [11], the authors analyze how relays are selected and conclude that almost $75 \%$ of them are selected in the first step of the greedy heuristic, so that improving the second step is not really useful. This conclusion seems correct, as long as the unit disk graph model is used.

\section{Physical Layer Model}

In the mathematical model defined in Section 2, the existence of a pair $(u, v) \in E$ is determined by the considered physical layer model and depends on several conditions, the most obvious one being the distance between $u$ and $v$. In the most commonly used model, known as the unit disk graph model, a bidirectional edge exists between two nodes if the distance between them is not greater than a given communication radius $R$ (it is assumed that all the nodes have the same communication radius). In this model, the set $E$ is then simply defined by:

$$
E=\left\{(u, v) \in V^{2}|u \neq v \wedge| u v \mid \leqslant R\right\},
$$

$|u v|$ being the Euclidean distance between nodes $u$ and $v$.

This model, while being widely spread, cannot be considered as realistic. Indeed, it is assumed that packets are always received without any error, as long as the distance between the transmitter and the receiver is smaller than the communication radius. This totally ignores random variations in the received signal strength, while it was demonstrated that their impact is really significant.

These fluctuations generate erroneous bits in the transmitted packets. If the error rate is sufficiently low, these bits can be repaired thanks to correction codes. However, if it is too high, then the packet must be dropped and a new transmission must be done. This supposes the existence of an acknowledgement mechanism (e.g., ACK packets) that cannot be used in broadcasting tasks due to the really high quantity of receivers. We thus assumed that no special mechanism is avail- 
able: we chose to locate our work at the application layer, solely based on link reliability. We believe that this is highly relevant to this research topic, because assuming that it could be very costly that packet loss is taken care of at a lower layer. The link reliability is simply equal to the probability of correct reception, which is influenced by a lot of factors (e.g., power of transmission, distance to the receiver(s), and presence of obstacles). We suppose in this paper that all the nodes have the same transmission radius, so that the power of transmission does not have to be considered here.

To consider the signal fluctuations, we thus change $G$ into a weighted graph where each edge $(u, v) \in E$ holds the probability $p(u, v)$ of correct reception between the two nodes $u$ and $v$. To mathematically model these probabilities, we chose to consider the lognormal shadowing model ${ }^{[2]}$. For this purpose, we used an approximated function $P(x)$ described in [12]:

$$
P(x)= \begin{cases}1-\frac{\left(\frac{x}{R}\right)^{2 \alpha}}{2}, & \text { if } 0<x \leqslant R \\ \frac{\left(\frac{2 R-x}{R}\right)^{2 \alpha}}{2}, & \text { if } R<x \leqslant 2 R \\ 0, & \text { otherwise }\end{cases}
$$

$\alpha$ being the power attenuation factor, and $x$ the considered distance. Fig.2 illustrates this model with $R=75$ and $\alpha=4$.

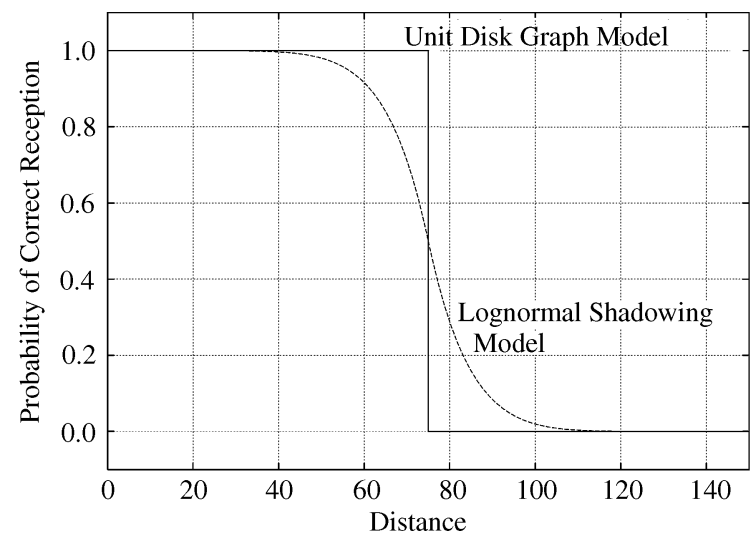

Fig.2. Unit disk graph and lognormal shadowing models $(R=75$, $\alpha=4)$.

We assume that each node $u$ is able to determine the probability $p(u, v)$ of correct reception of a packet that would be sent to a neighbor $v$. One simple way to gain this knowledge is to include a sequence number in each packet, and to let the nodes store for each neigh- bors the last $X$ sequence numbers received. To evaluate the link quality, the nodes simply have to subtract the oldest sequence number to the most recent one and to compare the resulting gap to $X$ : for instance, if the gap is $2 X$, then the link quality is equal to 0.5 . This value is updated each time a new packet is received, and may be included in HELLO messages sent by $v$ for $u$ to obtain the value of $p(u, v)$. For instance, we successfully implemented such method within the SensorScope project $^{1}$.

One of the major criticisms of the unit disk graph model is that it does not model the presence of obstacles between the nodes, and the lognormal shadowing model considers them neither, but we argue that it is sufficient enough for simulations. The most important factor is the weighting of edges by reception probabilities, the method used to distribute the latter is not important to compare protocols in general cases. A realistic model would be mandatory to simulate existing situations and to extract exact values. But in real cases, an obstacle would decrease the probability held by the corresponding edge and would thus be detected by the nodes when the sequence numbers are counted (if such method is considered). This means that in these cases, the broadcast algorithm would use "real" probabilities and its behavior would be adapted to that situation.

The unit disk graph and the lognormal shadowing models actually introduce two different behaviors.

- In the unit disk graph model, one has to maximize the length of each hop so that a single transmission may reach as many mobiles as possible: the quantity of needed transmitters is thus reduced.

- In the lognormal shadowing model, maximizing the length of each hop leads to smaller probabilities of correct reception, but minimizing them leads to a lot of energy consumption.

Some papers have already been published about routing in a realistic environment. Amongst them, DeCouto et al. ${ }^{[13]}$ and Draves et al. ${ }^{[14]}$ investigated the question of routing metrics for unicast protocols in wireless networks with a realistic physical layer: the key insight in most of this work is that hop-count based shortest-path routing protocols result in transmissions over long links. While this reduces the hop-count of routes, it also decreases the signal strength received at the receiver of these links, leading to very high loss rates and low end-to-end throughput. These papers also propose other routing metrics which incorporate link quality (e.g., in terms of error, congestion).

To the best of our knowledge, this paper is the first one to consider broadcasting over a realistic physical

(1) http://sensorscope.epfl.ch 
layer. We argue that broadcast fundamentally differs from unicast, and leads to a different trade-off between the length of each hop and the number of relays. Indeed, in a broadcast process, a node can rely on the redundancy introduced by the other transmitters. Further relays may thus be selected without decreasing the final delivery ratio. This is not possible in routing, as a given transmitter is the only one able to send the packet to the next hop. The redundancy of broadcasting must be fully considered in improving the performance of the underlying protocol.

\section{Evaluation of the Original MPR Algorithm}

In this section, we provide feedback about the performance evaluation we did with MPR and the lognormal shadowing physical model. We first describe our experimental setup, and then we present and analyze the results we obtained.

\section{$5.1 \quad$ Experimental Setup}

For our performance evaluation, we chose not to use a general purpose simulator in order to focus on the area of our study: we thus implemented the algorithms and the models in our own simulator. We first had to decide how to generate "realistic" graphs considering our realistic physical model. We chose to consider the method based on the sequence numbers (refer to Section 4) in conjunction with HELLO messages. That is, neighborhood information is stored in a table which is regularly cleaned in order to remove deprecated entries. An entry is deprecated when the corresponding node has not signaled itself in a given amount of time, that we denote by $x$. Beacon messages are regularly sent by each host to signal itself. Let us denote by $y$ the time between two HELLO messages (we have $x>y$ ). A node $u$ sees a neighbor $v$ if it has received at least one HELLO message during the last $y$ seconds. The probability $p_{n}(u, v)$ for this event to occur is equal to:

$$
p_{n}(u, v)=1-\overline{p(u, v)^{\frac{x}{y}}} .
$$

For each directional edge, a random number is thus drawn to determine if it exists. This way, when a node $u$ is aware of the existence of a neighbor $v$, it can decide to send messages to the latter. Of course, $u$ cannot be ensured that its messages will reach $v$ since they may get lost due to the channel randomness. Fig.3 illustrates this model with $x=1$ and $y=3$. One can easily observe that long edges have a high probability to be unidirectional while short edges have a high probability to be bidirectional.

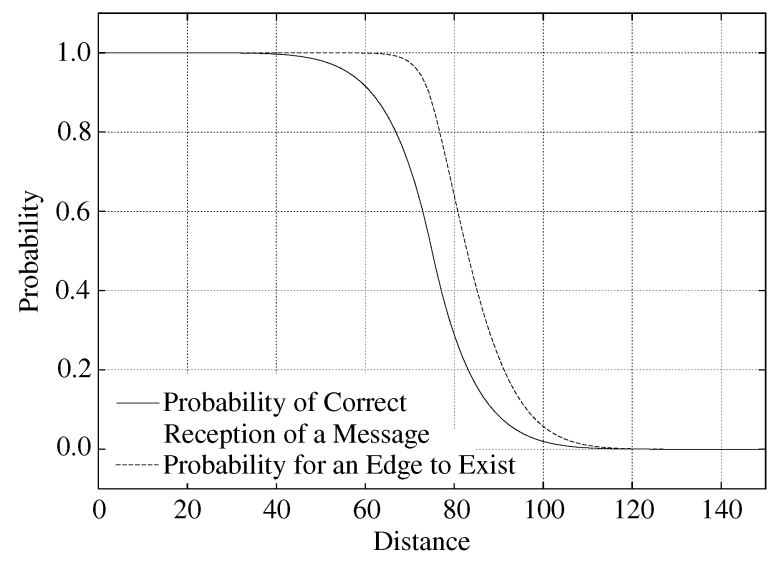

Fig.3. Impact of our realistic physical layer on edges $(x=1, y=$ $3, R=75)$.

All the results we present through the rest of this paper were obtained with the following parameters. The network is static and always composed of 500 nodes randomly distributed in a uniform manner over a square area whose size is computed in order to obtain a given average density. The edges are created using the method previously described, and for each measure, we took the average value of 500 iterations. We fixed the communication radius to be equal to $75 \mathrm{~m}$ in both physical models. An ideal MAC layer is considered to isolate the intrinsic properties of the selected relays: collisions of packets could skew both results and analyses.

\subsection{Experimental Results}

We provide in Fig.4(a) the delivery ratio of MPR for the two considered physical layers. When the unit disk graph model is used, a total coverage of the network is achieved as MPR is a deterministic algorithm. However, this is no longer the case with the lognormal shadowing model due to the multiple errors of transmission: the delivery ratio is under $75 \%$ for all considered density, and it is as low as $55 \%$ for the density $d=15$.

This poor performance can be explained by the fact that, as highlighted by Busson et al. in [11], the chosen relays are located at the limit of the communication range, where the probability of correct reception is very low. It is confirmed in our experiments, as illustrated in Fig.5. The average distance between a node and its multipoint relays is almost equal to $68 \mathrm{~m}$, while the maximal communication range is $75 \mathrm{~m}$. Moreover, [11] also states that $75 \%$ of the relays are chosen during the first step, which means that, when a relay does not correctly receive the message, there is a risk of $75 \%$ that this relay was the only one able to reach a given isolated node. The latter will thus not receive the message, potentially leading to a partition of the network. 


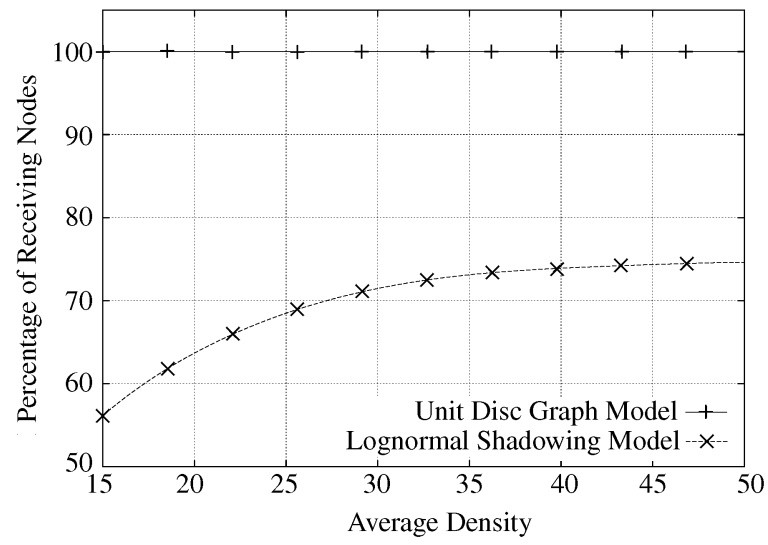

(a)

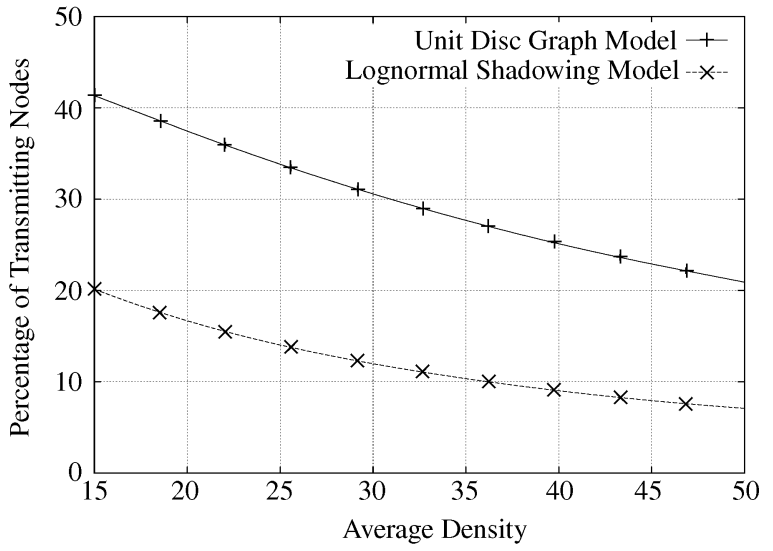

(b)

Fig.4. Performance of MPR with the two considered physical models. (a) Receiving nodes. (b) Transmitting nodes.

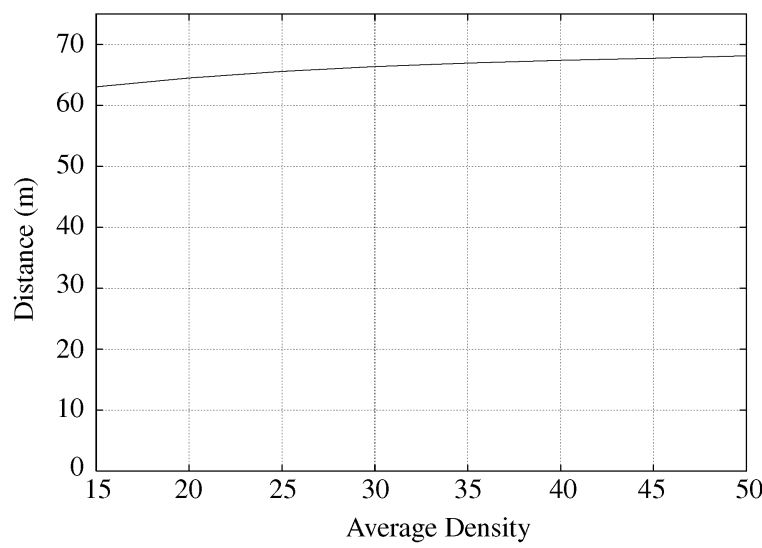

Fig.5. Average distance between a node and its MPR's.

We also provide in Fig.4(b) the percentage of nodes which correctly received and then relayed the message. It is interesting to note that this percentage is different for the two models. Indeed, as the only nodes which received the message are taken into account, one could have expected to observe the same values in both cases. This means that the needed number of relaying nodes does not linearly vary with the number of covered nodes: obviously, only a few relays are needed to cover a large number of different nodes, but a larger number is needed to cover the last few remaining ones.

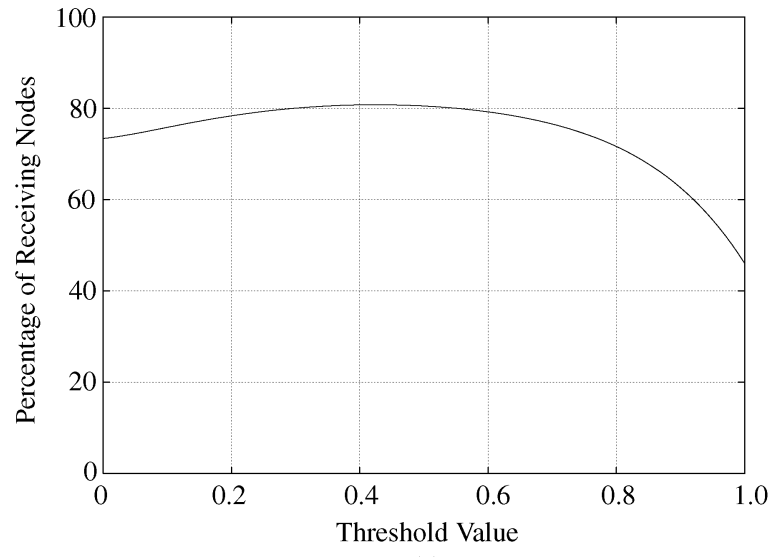

(a)

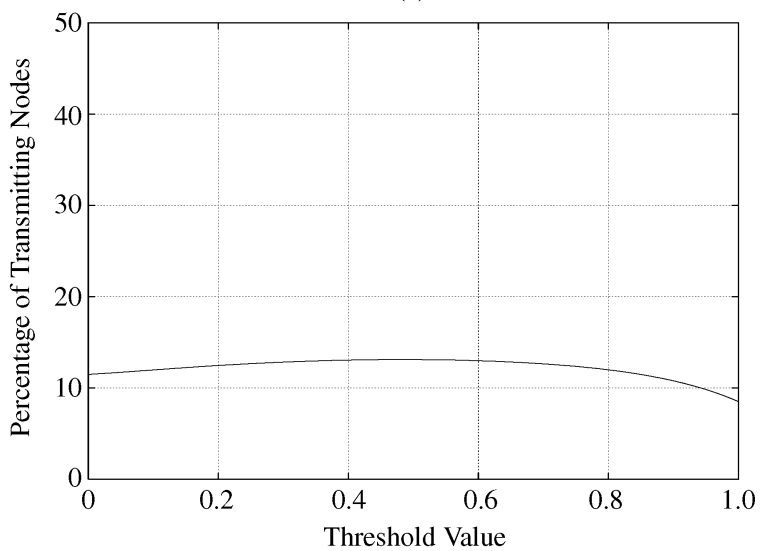

(b)

Fig.6. Performance of MPR broadcasting when simply removing lossy links (density $d=30$ ). (a) Receiving nodes. (b) Transmitting nodes.

In the routing protocol OLSR, lossy links are merely removed thanks to a threshold ${ }^{[15]}$. Simply, 1-hop neighbors that are reachable through a link of bad quality (i.e., which probability of correct reception is under the threshold) are not considered as potential multipoint relays. We implemented this straightforward solution and show the results in Fig.6. One can observe that while this solution may provide a slightly better delivery ratio with a correctly chosen threshold, the results are still not so good for a broadcasting protocol. With a low threshold, lossy links are considered and a bad delivery is observed. With a too high threshold, there are not enough possible relays to reach all 2-hop neighbors, and a bad delivery is observed once again. In the extreme case, where a threshold equal to 1.0 is used, no relays are selected in addition to the mandatory 1-hop 
neighbors.

The reason why this simple strategy does not work well is that it does not take into account the natural redundancy introduced by broadcasting. This redundancy must be really considered to keep a high delivery ratio when working with lossy links. This is what we do in the next section, where we propose original strategies to improve MPR broadcasting.

\section{Improving the Delivery of MPR Broadcasting}

As illustrated in the previous section, the original greedy heuristic used by Quayyum et al. in [3] is not suitable for a realistic physical layer. An average delivery of $75 \%$ is indeed not sufficient for most applications, and alternative solutions must be considered. We propose in this section some solutions to improving MPR broadcasting in a realistic environment. We keep notations introduced in Section 3. Thus, considering a node $u$, the set $\operatorname{MPR}(u)$ contains the multipoint relays chosen by $u$, while the set $M P R^{\prime}(u)$ contains 2-hop neighbors of $u$ not yet covered.

\subsection{New MPR Selection Strategies}

In this subsection, we propose miscellaneous strategies of improving the original greedy heuristic and to get back correct broadcasting performance. These strategies aim at maximizing the delivery ratio while keeping low the number of needed relays (and thus the energy consumption). In the following, the first step of the original heuristic, which allows isolated 2-hop neighbors to be covered, is kept since it is mandatory when aiming at fully covering the network.

\subsubsection{The First Strategy}

As previously explained, the low delivery ratio of MPR is caused by the high distance between a node and its relays. With the probability for the latter to correctly receive the broadcast packet being low, it is very likely that they will not be able to relay this packet. The 2-hop neighborhood of the transmitter is in this case not completely covered, resulting in a low delivery.

A first and straightforward strategy could be, when choosing a relay, to balance the coverage it provides and its probability to correctly receive the packet. Thus, in each step considering a node $u$, a score may be computed for each potential relay $v$. The neighbor with the highest score is selected and placed in $\operatorname{MPR}(u)$. We denote by $c_{u}(v)$ the additional coverage provided to $u$ by $v$ :

$$
c_{u}(v)=\left|\operatorname{MPR}^{\prime}(u) \cap N(v)\right| .
$$

The score obtained by $v$ at a given iteration for a node $u$, denoted by $s_{u}(v)$, is thus defined by:

$$
s_{u}(v)=c_{u}(v) \times p(u, v) .
$$

In simple terms, the additional coverage offered by $v$ is weighted by its probability to correctly receive the broadcast packet. In the original algorithm, we merely have $s_{u}(v)=c_{u}(v)$. This strategy is illustrated by Fig.7. Considering $u$, node $v_{1}$ covers three 2-hop neighbors $\left(w_{1}, w_{2}\right.$ and $\left.w_{3}\right)$. Its score $s_{u}\left(v_{1}\right)$ is thus equal to $3 \times p\left(u, v_{1}\right)$.

\subsubsection{The Second Strategy}

The previous strategy, while being more suitable for a realistic environment than the original one, still has an obvious flaw: it takes into account additional coverage in a too simple way. One can thus easily imagine a situation where a very distant 1-hop neighbor would offer a really high additional coverage, such that the latter would compensate a low probability of correct reception. In this case, this neighbor would be selected as a relay while its probability of correct reception, and thus being able to relay it, would be very low. One could also imagine a situation where the distance between the relay and the 2-hop neighbors it covers would be very long, such that the retransmission of the packet would have little chance to reach these 2-hop neighbors.

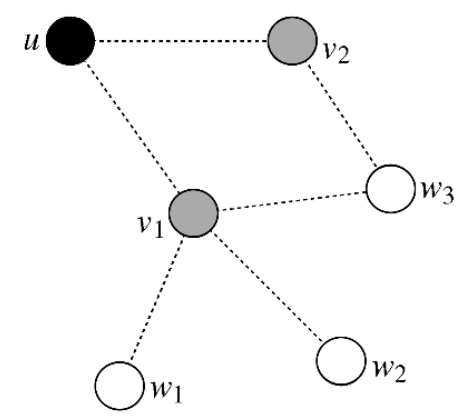

Fig.7. Case in which the node $u$ has to select its multipoint relays between its neighbors $v_{1}$ and $v_{2}\left(M P R(u)=\emptyset, \operatorname{MPR}^{\prime}(u)=\right.$ $\left.\left\{w_{1}, w_{2}, w_{3}\right\}\right)$.

We thus propose extending the concept used in the first proposal, by taking into account the probabilities of correct reception between the potential relay and the 2-hop neighbors it covers. We thus replace the additional coverage offered by a relay with the average probability of correct reception by the 2-hop neighbors. We thus obtain:

$$
s_{u}(v)=p(u, v) \times \sum_{i=1}^{i=\left|c_{u}(v)\right|} \frac{p\left(v, w_{i}\right)}{\left|c_{u}(v)\right|} .
$$


This way, opposite to the previous proposal, 1-hop neighbors offering a low coverage in terms of probabilities have little chance to be selected as multipoint relays. For instance in Fig.7, the score $s_{u}\left(v_{1}\right)$ of $v_{1}$ is now equal to $p\left(u, v_{1}\right) \times\left(\left(p\left(v_{1}, w_{1}\right)+p\left(v_{1}, w_{2}\right)+p\left(v_{1}, w_{3}\right)\right) / 3\right)$.

\subsubsection{The Third Strategy}

An even more clever strategy may actually consist in using the probability for all 2-hop neighbors to correctly receive the message from the considered multipoint relay. In the previous strategy, we indeed only consider the average probability while it might be preferable to consider the worst case. In this case, the score would then be equal to:

$$
s_{u}(v)=p(u, v) \times \prod_{i=1}^{i=\left|c_{u}(v)\right|} p\left(v, w_{i}\right) .
$$

For instance in Fig.7, the score $s_{u}\left(v_{1}\right)$ of $v_{1}$ would be equal to $p(u, v 1) \times p\left(v_{1}, w_{1}\right) \times p\left(v_{1}, w_{2}\right) \times p\left(v_{1}, w_{3}\right)$.

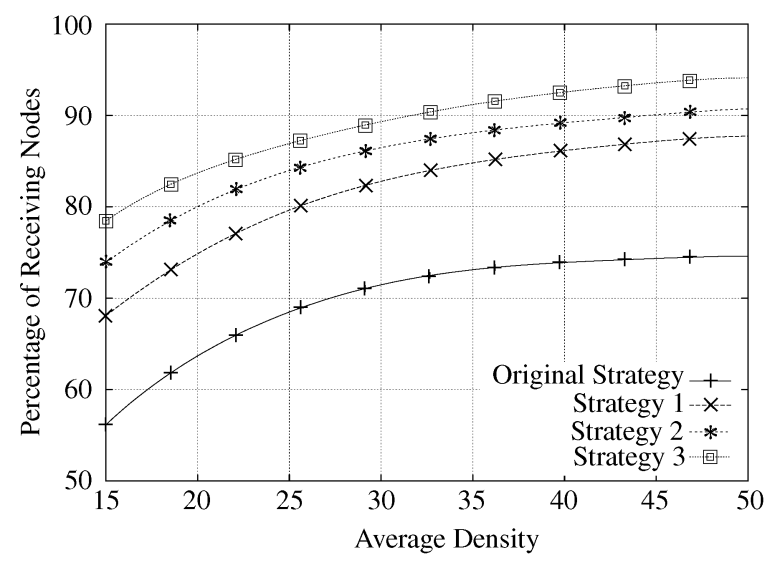

(a)

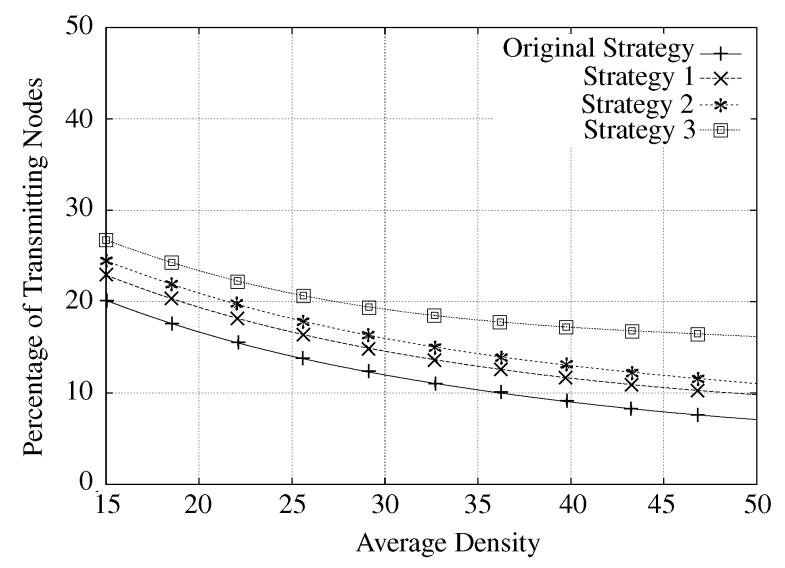

(b)

Fig.8. Performance of the different strategies with the lognormal shadowing model. (a) Receiving nodes. (b) Transmitting nodes.

\subsubsection{Performance Evaluation}

We start with Fig.8 which shows how well our strategies perform under the realistic assumptions of the lognormal shadowing model. Not surprisingly, we observe in Fig.8(a) that the new strategies lead to a far better delivery ratio than the original algorithm. This improvement is of course due to the use of the probabilities of correct reception given by the physical model. As expected, the third strategy offers a higher percentage of covered nodes simply because it prevents too far neighbors from being selected as relays. Considering the density $d=30$, the original strategy only covers $72 \%$ of nodes, against $83 \%, 86 \%$ and $89 \%$ for our three new strategies. The delivery ratio has thus been greatly improved, which was the main goal of our proposals.

As illustrated by Fig.8(b), the third strategy requires the participation of $19 \%$ of the receiving nodes for the density $d=30$ to provide a delivery ratio of $89 \%$. As a comparison, the second strategy uses only $16 \%$ of them to provide a delivery ratio of $86 \%$ at the same density. This is explained by the fact that the worst case is taken into account in the third strategy, leading to a slightly higher quantity of relays.

\subsection{Improving the Robustness}

While the delivery ratio of MPR broadcasting is greatly improved thanks to our new MPR selection strategies, it is still low in the lowest densities as illustrated in Fig.8(a). This may be explained by the fact that, as soon as a 2-hop neighbor has a non-null probability to be covered, it is removed from $M P R^{\prime}(u)$ and no longer considered. This removal is done even when the probability is very low, which in this case may be meaningless. It could be more interesting to remove a node from the non-covered list of 2-hop neighbors only when its probability to correctly receive the broadcast packet is sufficiently high, in order to increase the delivery ratio. For instance, a threshold may be used to define what "sufficiently high" means.

We thus propose keeping the strategies previously presented, while modifying the way 2 -hop neighbors are removed from $M P R^{\prime}(u)$. For such a 2-hop neighbor $w$ of $u$, its removal from $M P R^{\prime}(u)$ is done only if its coverage level $t_{u}(w)$ is over a given threshold. The value of $t_{u}(w)$ is given by the following:

$$
t_{u}(w)=1-\prod_{i=1}^{i=|M P R(u)|} \overline{p\left(v_{i}, w\right)}
$$

In simple terms, the coverage level of a 2-hop neighbor is equal to its probability to correctly receive the packet from at least one of the chosen relays. 
As an example, considering Fig.7, if the nodes $v_{1}$ and $v_{2}$ are selected as relays then the coverage level $t_{u}\left(w_{3}\right)$ of $w_{3}$ is equal to $1-\left(\overline{p\left(v_{1}, w_{3}\right)} \times \overline{p\left(v_{2}, w_{3}\right)}\right)$. Node $w_{3}$ is then removed from $\operatorname{MPR}^{\prime}(u)$ if $t_{u}\left(w_{3}\right)$ is over a given threshold. This way, several relays may thus now be selected to cover the same set of 2-hop neighbors, in order to increase the delivery ratio to a sufficient high level.

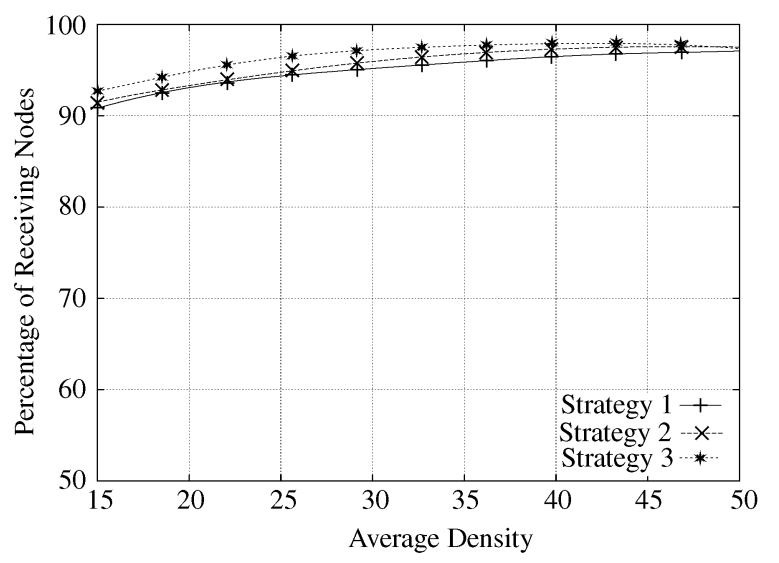

(a)

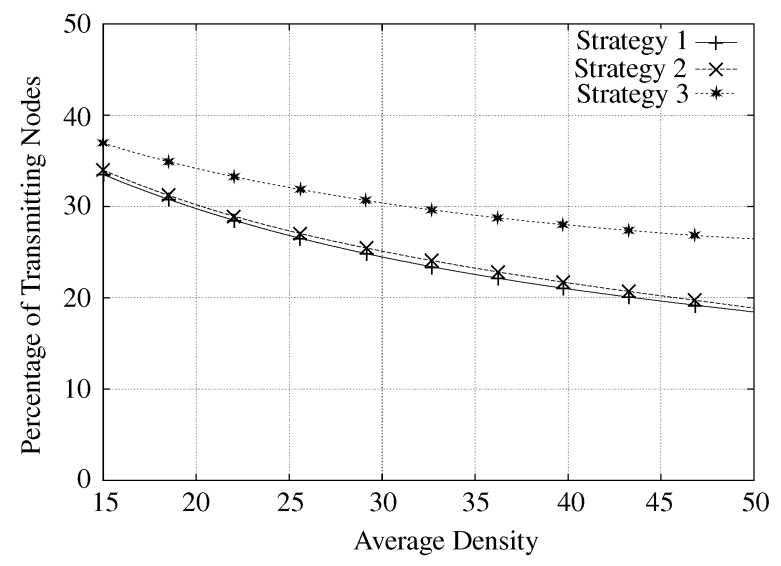

(b)

Fig.9. Performance of the new strategies with increased robustness (threshold is set to 0.3). (a) Receiving nodes. (b) Transmitting nodes.

We provide in Fig.9 the performance of all the previously presented strategies with this new "robustness" rule. The threshold is a fix value set to 0.3 . With this new rule, all strategies are able to provide a delivery ratio above $90 \%$, even in the lowest densities. As illustrated by Fig.8(b), achieving this delivery requires the participation of a higher quantity of relays because of the redundancy introduced to increase the robustness. This may seem to be a high value compared to previous figures, but considering the results given in Fig. 4 with the unit disk graph model, one can observe that values are almost the same for the original strategy. Indeed, almost $30 \%$ of relays are used by MPR with the unit disk graph at the density $d=30$. This means that the quantity of chosen multipoint relays for a given node is approximately the same with our new strategies and the robustness rule, but their choice is of better quality. Only a low level of redundancy is introduced for preserving a correct delivery ratio.

\section{Conclusion}

From the variety of results presented, we can observe that a realistic physical layer leads to miscellaneous problems while broadcasting. The MPR protocol is a good example: while being very efficient with the unit disk graph, its delivery ratio is not sufficient for most applications with a realistic model. While this study focuses on MPR, we believe that other main broadcasting methods, such as dominating sets, will exhibit the same flaws. However, some small modifications, which take into account the probabilities of correct reception, may correct these flaws. Thus, the new selection strategies that we presented for MPR does not modify the core of the protocol, only the selection process of multipoint relays is modified. One of the key aspects of our solutions is that they do not need any additional hardware, and may be implemented at the application layer without impacting on the whole communication stack. The results we presented showed that our solutions are efficient and really better than the original selection strategy.

Regarding open issues, some additional work may be done about the threshold of robustness used in Subsection 6.2 . Indeed, since the delivery ratio is not constant for all densities, an open issue relates to the way the threshold could be defined on the basis of the current density. It could be indeed interesting to use a higher threshold in lower densities to maximize the delivery, while using a lower threshold in higher densities could decrease the quantity of relays while achieving a correct delivery. From a more general point of view, some work is left to be done about the subject of broadcasting under realistic assumptions. As previously stated, we believe that most of the other well-known broadcasting protocols will need to be modified in order to keep correct performance while minimizing the energy consumption. In this quest for the good trade-off between robustness and efficiency, a mechanism such as the neighbor elimination scheme ${ }^{[16]}$ may be of prime importance. It is indeed able to retransmit packets only when needed, but may require to be adapted to realistic environments since nodes may believe that a packet has been lost while it was correctly received, leading to useless duplicated packets. There is also an open is- 
sue about neighborhood discovery: almost all localized protocols assume and require a correct knowledge of the neighborhood of nodes. Determining how to correctly populate neighborhood tables may be a whole research area by itself, since incorrect knowledge may lead to very bad behavior and performance.

\section{References}

[1] Ni S Y, Tseng Y C, Chen Y S, Sheu J P. The broadcast storm problem in a mobile ad hoc network. In Proc. the International Conference on Mobile Computing and Networking (MobiCom'99), Seattle, USA, August 1999, pp.151-162.

[2] Stojmenović I, Nayak A, Kuruvila J. Design guidelines for routing protocols in ad hoc and sensor networks with a realistic physical layer. IEEE Communications Magazine, March 2005, 43(3): 101-106.

[3] Qayyum A, Viennot L, Laouiti A. Multipoint relaying for flooding broadcast messages in mobile wireless networks. In Proc. the Hawaii International Conference on System Sciences (HICSS'02), Big Island, Hawaii, January 2002, pp.298307.

[4] Quin L, Kunz T. On-demand routing in MANETs: The impact of a realistic physical layer model. In Proc. the International Conference on Ad-Hoc, Mobile, and Wireless Networks (ADHOC-NOW'03), Montreal, Canada, October 2003, pp.37-48.

[5] Ingelrest F, Simplot-Ryl D. Maximizing the probability of delivery of multipoint relay broadcast protocol in wireless ad hoc networks with a realistic physical layer. In Proc. the International Conference on Mobile Ad-hoc and Sensor Networks (MSN 2006), Hong Kong, China, pp.157-168.

[6] Ingelrest F, Simplot-Ryl D, Stojmenović I. Resource Management in Wireless Networking. Chapter 17, Energy-Efficient Broadcasting in Wireless Mobile Ad Hoc Networks, Cardei M, Cardei I, Du D Z (eds.), Kluwer, 2004, pp.543-582.

[7] Jacquet P, Mühlethaler P, Clausen T, Laouiti A, Qayyum A, Viennot L. Optimized link state routing protocol for ad hoc networks. In Proc. the IEEE International Multi-Topic Conference (INMIC'01), Lahore, Pakistan, December 2001, pp.63-72.

[8] Adjih C, Jacquet P, Viennot L. Computing connected dominated sets with multipoint relays. Ad Hoc \& Sensor Wireless Networks, January 2005, 1(1/2): 27-39.

[9] Garey M R, Johnson D S. Computers and Intractability: A Guide to the Theory of NP-Completeness. W.H. Freeman, 1979.

[10] Lovasz L. On the ratio of optimal integral and fractional covers. Discrete Mathematics, 1975, 13: 383-390.

[11] Busson A, Mitton N, Fleury E. An analysis of the multi-point relays selection in OLSR. Technical Report 5468, INRIA, January 2005.

[12] Kuruvila J, Nayak A, Stojmenović I. Hop count optimal position based packet routing algorithms for ad hoc wireless networks with a realistic physical layer. In Proc. the IEEE International Conference on Mobile Ad Hoc and Sensor Systems (MASS 2004), Fort Lauderdale, USA, October 2004, pp.398405.

[13] DeCouto D, Aguayo D, Bicket J, Morris R. A high-throughput path metric for multi-hop wireless routing. In Proc. the Annual International Conference on Mobile Computing and
Networking (MobiCom'03), San Diego, USA, September 2003, pp.134-146.

[14] Draves R, Padhye J, Zill B. Routing in multi-radio, multi-hop wireless mesh networks. In Proc. the ACM SIGCOMM 2004, Portland, USA, September 2004, pp.114-128.

[15] Laouiti A, Adjih C. Mesures des performances du protocole OLSR. In Proc. the IEEE International Conference on Sciences of Electronic, Technology of Information and Telecommunications (SETIT 2003), Sousse, Tunisia, 2003, pp.265278.

[16] Stojmenović I, Seddigh M. Broadcasting algorithms in wireless networks. In Proc. the SSGRR, International Conference on Advances in Infrastructure for Electronic Business, Science, and Education on the Internet, L'Aquila, Italy, July 2000 .

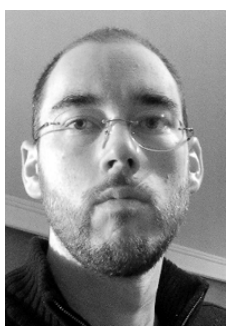

François Ingelrest received the Master's degree from the University of Lille, France, in 2003. He did his Ph.D. thesis at the Fundamental Computer Science Laboratory of Lille (LIFL), France, in the POPS research group, which is specialized in operating systems and networking for small portable objects. He is now a postdoctoral researcher at the Audiovisual Communications Laboratory, EPFL, Switzerland. His main research axis is about communication protocols for wireless ad hoc and sensor networks.

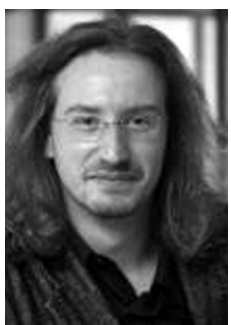

David Simplot-Ryl received the Graduate Engineer degree in computer science, automation, electronic and electrical engineering, the M.Sc. and Ph.D. degrees in computer science from the University of Lille, France, in 1993 and 1997, respectively. In 1998, he joined the Fundamental Computer Science Laboratory of Lille (LIFL), France, where he is currently associate professor. He received the Habilitation degree from University of Lille, France, in 2003. His research interests include ad-hoc networks, distributed computing, embedded operating systems and RFID technologies. Recently, he mainly contributes to international standardization about RFID tag identification protocols in partnership with Gemplus and TagSys companies. He is currently associate editor of International Journal of Computers and Applications (IASTED/Acta Press). He is also guest editor of special issues of IEEE Network Magazine (IEEE Communication Society), and Ad Hoc Networks Journal (Elsevier). He is also co-chair for a workshop at IEEE Int. Conf. Distributed Computing and Systems ICDCS 2004. He is leader and scientific director of the several research groups and projects of the University of Lille, IRCICA research institute and INRIA research institute. Since 2007, he is research vice-chair of INRIA Futurs Research Centre. 